\title{
MAPAS TEMÁTICOS DE COMPONENTES PRINCIPAIS PARA CARACTERIZAÇÃO DE PROPRIEDADES QUÍMICAS DO SOLO
}

\author{
Márcio Paulo de Oliveira ${ }^{1}$, Gustavo H. Dalposso ${ }^{2}$, Carlos Roberto Espires ${ }^{3}$, Jessé Fernando Faria ${ }^{4}$
}

\section{RESUMO}

A agricultura na região Oeste do Paraná tem destaque por intensa atividade agrícola, na qual busca altas produtividades. A produtividade das áreas de cultivo está diretamente relacionada com a fertilidade. O uso intensivo do solo tende à acidificação e diminuição nos teores de nutrientes nele armazenados, o que, por consequência, limita também o desenvolvimento radicular, contribuindo para redução da produtividade. O presente trabalho foi desenvolvido com o intuito de investigar a relação entre as propriedades químicas do solo: acidez potencial, cálcio, magnésio, potássio, pH do solo, teor de matéria orgânica, soma de bases, capacidade de troca de cátions total e saturação por bases, na camada de 0,0 a $0,20 \mathrm{~m}$. A exploração do solo na região se faz de forma intensiva com cultivos de soja, milho, trigo e feijão em sucessão de culturas. A amostragem de solo para análise das referidas variáveis foi georreferenciada ( $g r i d$ de 2 ha), sendo utilizadas técnicas de geoestatística e análise de componentes principais, com o intuito de identificar regiões que possuam maior acidez e deficiência de nutrientes que possam ser supridos por meio de calagem. Os resultados permitiram evidenciar a relação da acidez do solo com variáveis como cálcio e magnésio o que sugere a correção desses nutrientes nas regiões de elevada acidez. A classificação dos mapas em classes permite o direcionamento de ações em campo nas unidades com características similares, sendo importante para o trato localizado destas subregiões na área de estudo.

Palavras-chave: fertilidade do solo, análise multivariada, variabilidade espacial.

\section{ABSTRACT \\ THEMATIC MAPS OF PRINCIPAL COMPONENTS FOR CHARACTERIZATION OF SOIL CHEMICAL PROPERTIES}

The western region of Paraná is known for its intensive agricultural activity seeking high productivity. Productivity of crop cultivation areas is directly related to soil fertility, and intensive soil use gives rise to acidification and a reduction in nutrient levels, in turn limiting root development and contributing to reduced productivity. This study was conducted with the aim of investigating relationships between the following chemical soil properties: potential acidity; calcium; magnesium; potassium; soil pH; organic matter; sum of bases; total cation exchange capacity and base saturation in the layer from 0.0 (zero) to $0.2 \mathrm{~m}$. Soil use in the region is intensive, with cultivation of soybeans, corn, wheat and beans. Soil sampling for parameter analysis was georeferenced (on a grid of 2 ha), and geostatistical techniques and principal component analysis were employed to identify regions of highest soil acidity and nutrient deficiency treatable by liming at different levels. The results showed the relationship between soil acidity with variable such that calcium and magnesium which indicates the need to fix these nutrients in the soil in areas with higher acidity. The maps have five classes that allow us to select sub regions in study area to field actions, being important to localized treatment in the area.

Keywords: soil fertility, multivariate analysis, spatial variability.

\section{Recebido para publicação em 09/09/2014. Aprovado em 17/11/2015.}

1 - Matemático, M. Sc., Professor Assistente da UTFPR/Toledo-PR, marciooliveira@utfpr.edu.br

2 - Matemático, M. Sc., Professor Assistente da UTFPR/Toledo-PR, gustavodalposso@utfpr.edu.br

3 - Agrônomo, PLANTAR/Cascavel-PR, carlos@plantarnet.com.br

4 - Agrônomo, PLANTAR/Cascavel-PR, jesse@plantarnet.com.br 


\section{INTRODUÇÃO}

O sistema de plantio direto (SPD) auxilia na manutenção da palha sobre o solo, no controle de erosão, na conservação da umidade do solo, no aumento da matéria orgânica e na atividade biológica, o que contribui para a estabilidade dos agregados (AMADO et al., 2007). Esse sistema permite uma redução dos custos de produção, comparativamente ao sistema convencional, pois não há operações de preparo periódico do solo relacionada a etapa de semeadura, de modo que o tempo de trabalho e o consumo de combustível são reduzidos.

Nas áreas sob SPD geralmente observa-se o problema de compactação do solo, pois, segundo Tormena et al. (1998), no SPD, a movimentação do solo é restrita à linha de semeadura, mas a ocorrência sistemática do tráfego causa compactação na camada superficial do solo. A intensidade da compactação depende de fatores como a sequência de culturas, do sistema de manejo e da umidade do solo por ocasião do tráfego de máquinas (CELIK et al., 2011).

O SPD é um sistema que possui uma elevada complexidade e que também apresenta variabilidade espacial significativa de atributos físicos e químicos do solo. Assim, como nos demais sistemas de cultivo, no SPD, o conhecimento dessa variação é importante para o levantamento, manejo do solo e gerenciamento de práticas agrícolas (SILVA et al., 2010).

A fertilidade do solo é um fator importante que, em baixos níveis, afeta o desenvolvimento da soja e influencia negativamente o rendimento dos grãos (THOMAS et al., 1998).

$\mathrm{O} \mathrm{pH}$ fornece indícios das condições químicas gerais de um solo. $\mathrm{O}$ pH indica a predominância de íons hidrogênio $\left(\mathrm{H}^{+}\right)$que existe no solo, sendo que em um solo é ácido quando possui muitos íons $\left(\mathrm{H}^{+}\right)$ e poucos íons de cálcio $\left(\mathrm{Ca}^{2+}\right)$, magnésio $\left(\mathrm{Mg}^{2+}\right)$ e potássio $\left(\mathrm{K}^{+}\right)$em seu complexo de troca.

A acidez potencial trocável é aquela que está relacionada com os íons $\mathrm{Al}^{3+}$ e $\mathrm{H}^{+}$retidos na superfície dos coloides do solo, e como a quantidade de íons $\mathrm{H}^{+}$é pequena (menos de $5 \%$ da acidez trocável), temos que a acidez trocável é uma medida da acidez no solo.

Solos ácidos são comuns nas regiões sob condições tropicais, onde a grandeza da precipitação pluviométrica é tal que os elementos alcalinos, como $\mathrm{Ca}^{2+}$ e o $\mathrm{Mg}^{2+}$, são lixiviados das camadas superiores pelas (EMBRAPA, 2010).

A análise da estrutura da dependência espacial da fertilidade dos solos requer que muitos nutrientes sejam analisados conjuntamente. Essa tarefa, realizada por meio de métodos estatísticos univariados, eleva a dimensão do problema e onera o processo de análise (SILVA et al., 2010).

Uma opção para tais tipos de dados é o uso de técnicas de análise multivariada, como a análise de componentes principais (ACP), a fim de explicar a estrutura da variância e da covariância de um conjunto de variáveis por meio da construção de combinações lineares dessas e da redução dimensional do fenômeno em estudo (JOHNSON; WICHNER, 2007). A ACP é uma técnica multivariada de modelagem da estrutura de covariância, em que se busca determinar variáveis latentes que são representadas por combinações lineares de um grupo de variáveis subjacentes que são relacionadas entre si (FERREIRA, 2008).

As ACP são empregadas em vários estudos na agricultura, como, por exemplo, na avaliação do efeito da qualidade da irrigação em solos com diferentes propriedades químicas, físicas e biológicas (MANDAL et al., 2008), no estudo de perfis de solo com alto teor de material orgânica em diferentes regiões do Brasil (VALLADARES et al., 2008) e no estudo de relações de propriedades físicas e químicas do solo irrigado com água residuária (ADHIKARI et al., 2011).

Uma alternativa para a análise de dados que possuem estrutura de dependência espacial é por meio da elaboração de mapas temáticos, que é precedida de uma análise geoestatística entendida como o conjunto de procedimentos estatísticos que são aplicados em estudos cujos dados são referenciados no espaço, considerando não apenas o valor observado, mas também a característica de

\section{REVENG}


que observações mais próximas geograficamente tendem a possuir valores mais parecidos (RIBEIRO JUNIOR, 1995).

Assim, este trabalho teve por objetivo investigar a relação entre as propriedades químicas do solo: acidez potencial, cálcio, magnésio, potássio, $\mathrm{pH}$ do solo, teor de matéria orgânica, soma de bases, capacidade de troca de cátions total e saturação por bases, na camada de 0,0 a $0,20 \mathrm{~m}$ em um Latossolo Vermelho distroférrico típico cultivado em SPD, por meio da dependência espacial de propriedades químicas do solo, com o uso conjunto de técnicas de análise de componentes principais e geoestatística.

\section{MATERIAL E MÉTODOS}

A área em estudo está situada em Cascavel-PR na coordenada $24^{\circ} 42^{\prime} 39.00^{\prime \prime} \mathrm{S}$ e $53^{\circ} 28^{\prime} 57.00^{\prime \prime} \mathrm{O}$, com altura média de $615 \mathrm{~m}$, precipitação anual de $3324 \mathrm{~mm}$ e temperaturas médias anuais de 20,72 ${ }^{\circ} \mathrm{C}$. O clima local é temperado mesotérmico super úmido, segundo a Classificação de Köeppen. O solo da área estudada foi classificado como sendo um Latossolo Vermelho distroférrico típico, textura argilosa a muito argilosa com $740 \mathrm{~g} / \mathrm{kg}$ de argila, $140 \mathrm{~g} / \mathrm{kg}$ de silte e $120 \mathrm{~g} / \mathrm{kg}$ de areia, substrato basalto e relevo suave ondulado (EMBRAPA, 2006).

As variáveis químicas do solo consideradas foram a acidez potencial $(\mathrm{H}+\mathrm{Al})$, cálcio $(\mathrm{Ca})$, magnésio $(\mathrm{Mg})$ e potássio $(\mathrm{K}), \mathrm{pH}$, teor de matéria orgânica (MO), soma de bases (SB), capacidade de troca de cátions total (CTC) e saturação por bases (V\%).

A amostragem de solo foi realizada de $17 / 08 / 2012$ a 20/08/2012. Utilizou-se um GPS Trimble Juno $3 D$ para determinar o perímetro da área e localizar os pontos amostrais, que foram definidos segundo um grid regular formado por 45 pontos, a uma distância de $141 \mathrm{~m}$ entre pontos, sendo que cada ponto amostral é representante de 2 ha, conforme apresentado na Figura 1.

As Figuras 1A e 1B apresentam os grids de amostragem para a camada de 0,0 a $0,10 \mathrm{~m}$ e 0,10 a $0,20 \mathrm{~m}$, respectivamente, sendo que, para cada elemento amostral na camada de 0,0 a 0,10 $\mathrm{m}$, foram retiradas 9 subamostras para formar uma amostra composta representativa de 2 ha (Figura 1A). No mesmo grid foram retiradas, para cada elemento amostral, 2 subamostras na camada de 0,10 a $0,20 \mathrm{~m}$, para constituir uma amostra composta representativa de 12 ha, sendo que os últimos elementos amostrais, para obter equivalente representatividade, foram coletadas três subamostras por ponto para a composição da amostra composta representativa de 2 ha para cada ponto (Figura 1B).

As amostras estratificadas foram obtidas para investigar a variabilidade química dos atributos do solo, o qual apresenta maior variabilidade na horizontal, sendo que nesta direção a mesma pode ser induzida por praticas e processos de manejo em superfície (CORÁ, 2004). A variabilidade vertical reduz com a profundidade.

As amostras foram coletadas com amostrador SACI (Soluções em Amostragem e Coleta de Informações), acoplado em um quadriciclo Honda Fourtrac 4x4. O equipamento SACI Trail é composto por comando automático, com sensores de profundidade e $G P S$, garantindo a rastreabilidade do trabalho em campo, com opção de amostragem em camadas estratificadas.

O cálculo de calagem para a área foi realizado pela média da camada de 0,0 a $0,10 \mathrm{~m}$ e 0,10 a 0,20 m, pois, segundo EMBRAPA (2011), a necessidade de calagem é recomendada para a camada de 0,0 $0,20 \mathrm{~m}$. Por meio de um grid amostral, foram elaborados mapas temáticos utilizando métodos geoestatísticos para as variáveis químicas.

Uma análise multivariada de dados, considerando componentes principais PC1 e PC2, foi realizada. Por meio dos valores das variáveis químicas nos 45 elementos amostrais, foram calculados os valores das componentes principais PC1 e PC2, considerando os pesos que cada variável recebeu pela análise das componentes principais. Os mapas temáticos das PC1 e PC2 foram obtidos por krigagem, com o intuito de obter mapas de superfície com o uso da redução do número de variáveis. 


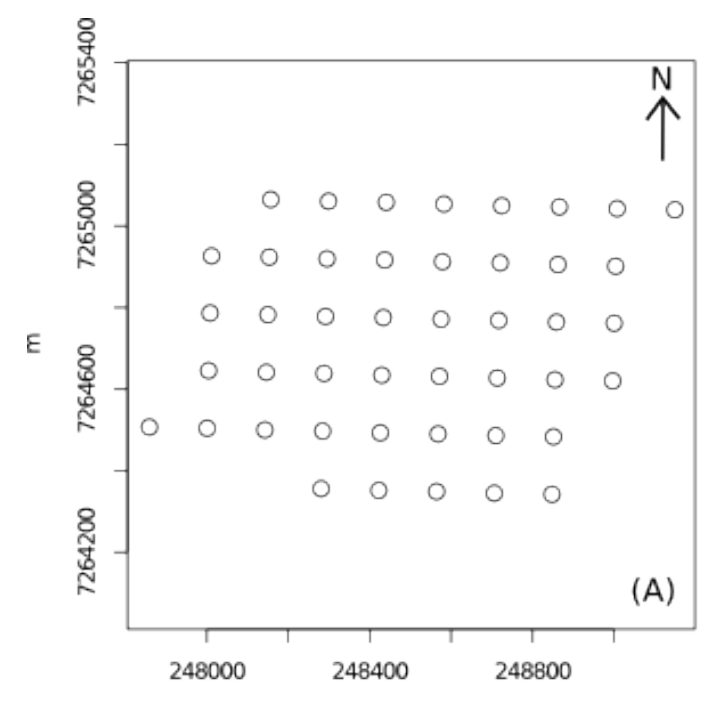

$m$

Elementos amostrais com distância de 141 metros, camada 0,0 a $0,10 \mathrm{~m}$, com nove sub amostras por elemento, para cada amostra composta de 2 ha.

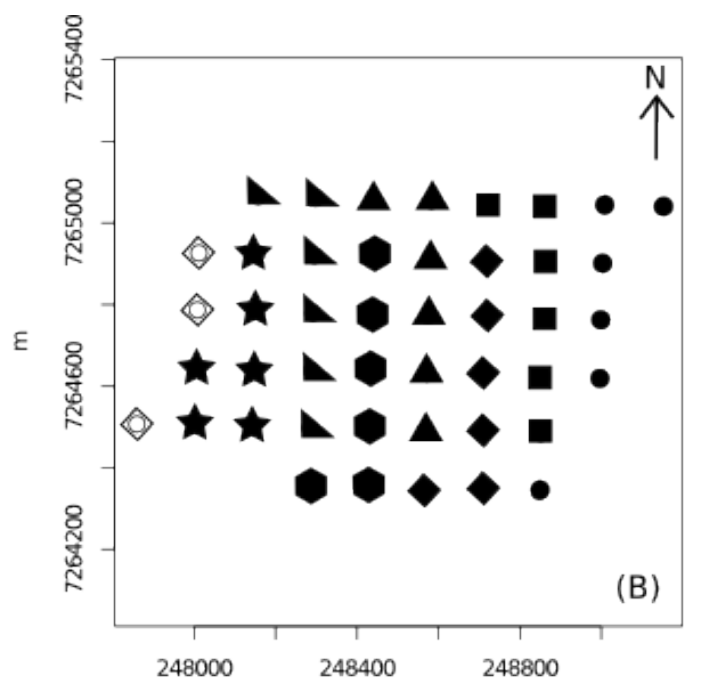

- $\Delta \Delta \Delta^{\mathrm{m}} \boldsymbol{\Delta}$ Elementos amostrais com distância de 141 metros, camada 0,1 a 0,20 m, com duas sub amostras por elemento, para cada amostra composta de 12 ha.

$\checkmark$ Elementos amostrais, camada 0,10 a $0,20 \mathrm{~m}$, com três sub amostras por elemento, para cada amostra composta de 6 ha.

Figura 1. (A) Grade amostral com 45 pontos considerando uma distância de $141 \mathrm{~m}$ entre pontos, camada de 0,0 a $0,10 \mathrm{~m}$; (B) Grade amostral com os 45 elementos na camada 0,10 a $0,20 \mathrm{~m}$.

A dependência espacial de uma variável regionalizada pode ser avaliada por meio do semivariograma experimental, cujas semivariâncias $\gamma(h)$ são definidas como a metade da esperança matemática do quadrado da diferença do valor da variável regionalizada $Z$, observada em dois pontos separados no espaço por um vetor $h$, sendo dada pela seguinte equação:

$$
\gamma(h)=1 / 2 E\left[(Z(x)-Z(x+h))^{2}\right]
$$

A função de semivariância $\gamma(h)$ pode ser estimada pelo estimador clássico de Matheron (1963), dado por:

$$
\hat{\gamma}(h)=\frac{1}{2 N(h)} \sum_{i=1}^{N(h)}\left[Z\left(x_{i}\right)-Z\left(x_{i}+h\right)\right]^{2}
$$

Em que, $\hat{\gamma}(h)$ é a semivariância estimada, $N(h)$ é o número de pares de dados $\ Z\left(x_{i}\right) Z\left(x_{i}+h \rrbracket\right.$ , utilizados na estimativa, e $h$ a distância entre as observações das variáveis aleatórias $Z\left(x_{i}\right)$.

A análise de componentes principais é uma técnica que permite explicar a estrutura de variânciacovariância de um vetor aleatório composto de $\mathrm{p}$ variáveis aleatórias, por meio de combinações lineares entre as variáveis originais. As combinações lineares são chamadas de componentes principais e são não correlacionadas entre si. Considerando $\mathrm{p}$ variáveis aleatórias, é possível se obter $\mathrm{p}$ componentes principais, no entanto, o objetivo da análise de componentes principais é reduzir a dimensão dos dados originais, com vistas a facilitar a identificação de padrões, permitindo a interpretação pela descoberta de relações, previamente não evidentes.

Embora seja necessária a utilização das $\mathrm{p}$ componentes principais para explicar a variabilidade total das variáveis, podemos utilizar um conjunto $\mathrm{k}$ menor de componentes principais com vistas à redução da dimensão dos dados originais e a facilitar a intepretação. Em geral, podemos substituir as $\mathrm{p}$ variáveis originais (considerando 80 a $90 \%$ da variância total das variáveis originais) por 1, 2 ou 3 componentes principais, sem muita perda de informação (JOHNSON; WICHERN, 2007).

\section{REVENG}

ENGENHARIA NA AGRICUlTURA, VIÇOSA - MG, V.23 N.6, NOVEMBRO / DEZEMBRO 2015 
Uma vez determinados os componentes principais de um conjunto multivariado, realizou-se a etapa de interpretação de seus coeficientes. Os coeficientes das variáveis nessa combinação, conforme sejam maiores em valor absoluto, indicam maior importância daquela variável na formação do componente, de modo positivo ou negativo, conforme o sinal do coeficiente. Essa avaliação dos coeficientes permite identificar contrastes entre variáveis com maiores valores de coeficientes no componente principal, positivo e negativos.

\section{RESULTADOS E DISCUSSÃO}

No Quadro 1 estão apresentadas as estatísticas descritivas dos valores das variáveis químicas do solo na profundidade de $0-0,20 \mathrm{~m}$. A variável $\mathrm{K}$ não apresentou normalidade a 5\% de significância pelos testes de Shapiro-Wilks e Kolmogorov-Smirnov. Essa variável apresentou pontos discrepantes que podem ter influenciado em sua normalidade. As variáveis $\mathrm{H}+\mathrm{Al}, \mathrm{Ca}, \mathrm{Mg}, \mathrm{pH}, \mathrm{MO}, \mathrm{SB}, \mathrm{CTC}$ e V\% apresentaram uma distribuição normal com os referidos testes. O conjunto multivariado não apresentou distribuição normal multivariada com os testes Shapiro-Wilks e Shapiro-Francia.

Segundo a classificação de Warrick e Nielsen (1980), os coeficientes de variação apresentaramse baixos $(<10 \%)$ para $\mathrm{H}+\mathrm{Al}, \mathrm{pH}, \mathrm{MO}$ e $\mathrm{CTC}$, médios (CV entre 10 e 20\%) para $\mathrm{Ca}, \mathrm{Mg}, \mathrm{K}, \mathrm{SB}$ e V, sendo que os valores elevados de CV podem ser considerados como os primeiros indicadores da existência de heterogeneidade nos dados (GOMES, 2000).

Os valores médios dos atributos químicos do solo foram classificados, segundo EMBRAPA (2013) para a região central do Brasil, como altos $\left(>0,8 \mathrm{cmol}_{\mathrm{c}} / \mathrm{dm}^{3}\right)$ para cálcio, alto $\left(>0,8 \mathrm{cmol}_{\mathrm{c}} /\right.$ $\left.\mathrm{dm}^{3}\right)$ para magnésio, muito alto $\left(>0,31 \mathrm{cmol}_{\mathrm{c}} / \mathrm{dm}^{3}\right)$ para o potássio, muito alto $\left(>12,0 \mathrm{mg} / \mathrm{dm}^{3}\right)$, alto $(>$ $5,0 \mathrm{cmol} / \mathrm{dm}^{3}$ ) para a acidez potencial e alto para o teor de MO \% (> 2,5 \%) (RIBEIRO et al., 1999). $\mathrm{O}$ valor médio do $\mathrm{pH}$ indica alta acidez do solo, o qual possui correlação positiva com a $\mathrm{V}$ a qual se deseja elevar no complexo de troca de cátions.

Os teores de $\mathrm{Ca}, \mathrm{Mg}$ e $\mathrm{K}$ apresentaram níveis altos, baixo e muito bom, respectivamente, na camada $0,0-0,20 \mathrm{~m}$, sendo que as suas relações médias foram de 2,42 para $\mathrm{Ca}: \mathrm{Mg}$, queé classificado como médio $(1,5-3,5), 10,4$ para $\mathrm{Ca}: \mathrm{K}$ médio $(8$ - 16) e de 4,3 para Mg:K, também classificado como médio (3 - 6), e, conforme EMBRAPA (2013), podem estar afetando a produtividade das culturas. Watanabe et al. (2005) verificaram que relações equilibradas dos elementos $\mathrm{Ca}, \mathrm{Mg}$ e $\mathrm{K}$, no complexo de troca, aumentaram a produtividade da cultura da soja de um Latossolo Distroférrico no Paraná.

Nogara Neto et al. (2011), em estudo sobre a relação de propriedades do solo na variação espacial

Quadro 1. Variáveis de estatísticas descritivas.

\begin{tabular}{lccccccccccc}
\hline & Min & Q1 & Med & Média & Q3 & Max & DP & CV $(\%)$ & A & C & N \\
\hline $\mathrm{H}+\mathrm{Al}\left(\mathrm{cmol}_{\mathrm{c}} / \mathrm{dm}^{3}\right)$ & 5,38 & 6,55 & 6,92 & 6,88 & 7,25 & 7,75 & 0,52 & 7,56 & $-0,62$ & 3,24 & Sim \\
$\mathrm{Ca}\left(\mathrm{cmol}_{\mathrm{c}} / \mathrm{dm}^{3}\right)$ & 3,29 & 3,88 & 4,09 & 4,16 & 4,37 & 5,28 & 0,48 & 11,6 & 0,49 & 2,93 & Sim \\
$\mathrm{Mg}\left(\mathrm{cmol}_{\mathrm{c}} / \mathrm{dm}^{3}\right)$ & 1,15 & 1,52 & 1,64 & 1,72 & 1,83 & 2,9 & 0,32 & 18,8 & 1,24 & 5,62 & Sim \\
$\mathrm{K}\left(\mathrm{cmol}_{\mathrm{c}} / \mathrm{dm}^{3}\right)$ & 0,29 & 0,36 & 0,41 & 0,4 & 0,44 & 0,51 & 0,06 & 14,3 & $-0,24$ & 2,1 & Não \\
$\mathrm{pH}$ & 4.78 & 4.91 & 5.00 & 5.01 & 5.11 & 5.35 & 0.14 & 2.89 & 0.25 & 2.32 & Sim \\
$\mathrm{MO}(\%)$ & 2.85 & 3.24 & 3.38 & 3.39 & 3.56 & 3.86 & 0.22 & 6.58 & -0.10 & 2.56 & Sim \\
$\mathrm{SB}\left(\mathrm{cmol}_{\mathrm{c}} / \mathrm{dm}^{3}\right)$ & 4.77 & 5.84 & 6.12 & 6.28 & 6.66 & 8.64 & 0.81 & 12.91 & 0.64 & 3.39 & Sim \\
$\mathrm{CTC}\left(\mathrm{cmolc}_{\mathrm{dm}}\right)$ & 12.00 & 12.90 & 13.10 & 13.20 & 13.50 & 14.00 & 0.43 & 3.26 & 0.00 & 2,93 & Sim \\
$\mathrm{V}(\%)$ & 39.30 & 44.8 & 46.8 & 47.6 & 50.0 & 61.6 & 4.90 & 10.30 & 0.57 & 3.25 & Sim \\
\hline
\end{tabular}

Min: mínimo; $\mathrm{Q}_{1}$ : primeiro quartil; Med: mediana; $\mathrm{Q}_{3}$ : terceiro quartil; Max: máximo; DP: desvio padrão; $\mathrm{CV}$ : coeficiente de variação; A: assimetria; C: curtose; N: Normalidade univariada com os testes de Shapiro-Wilks e Kolmogorov-Smirnov com 5\% de significância. 
de produtividade de grãos de milho, verificaram que o equilíbrio de bases foi importante para a nutrição de plantas de milho com implicações na produtividade. Estes autores relataram a importância da relação $\mathrm{Mg}: \mathrm{K}$, com valor crítico de 2,30, uma vez que a produtividade de milho decresceu quando apresentou menores valores.

Foram classificados como muito alto o valor médio da soma de bases, alto o valor da capacidade de troca de cátions total, e médio a saturação por bases (RIBEIRO et al., 1999). Os resultados para os valores médios dos teores de $\mathrm{Ca}, \mathrm{Mg}$ e $\mathrm{K}$, podem ser explicados pelo sistema de manejo adotado na propriedade, SPD com rotação de culturas.

No Quadro 2 apresentam-se as cargas (loadings) das duas componentes principais PC1 e PC2, que conjuntamente explicam $82,98 \%$ da proporção da variação nas variáveis $\mathrm{H}+\mathrm{Al}, \mathrm{Ca}, \mathrm{Mg}, \mathrm{K}, \mathrm{pH}$, $\mathrm{MO}, \mathrm{SB}, \mathrm{CTC}$ e $\mathrm{V}$, de modo que apenas essas componentes serão utilizadas, pois, segundo Johnson e Wichern (2007), as componentes principais que acumuladamente explicam $80 \%$ ou mais da variação nos dados é considerada adequada para a redução do número de variáveis em uma análise multivariada.

A acidez potencial tende a ser neutralizada na presença de íons $\mathrm{Ca}$ e $\mathrm{Mg}$, de modo que uma análise multivariada para identificar as relações de bases e ácidos é de interesse para uma busca do equilíbrio de nutrientes no solo.

A Figura 2 apresenta o gráfico biplot das componentes principais $\mathrm{PC} 1$ contra $\mathrm{PC} 2$, em que as correlações das variáveis químicas são evidenciadas. Nessa mesma figura, a componente PC1, que explica $69,74 \%$ da variação dos dados, apresentou positiva correlação com a acidez potencial $(\mathrm{H}+\mathrm{Al})$ e negativa com as demais variáveis, de modo que o efeito do fator de variação de $\mathrm{H}+\mathrm{Al}$ conduz a um incremento destes valores, enquanto que os valores das outras variáveis são reduzidos. Este componente pode ser interpretado como a resposta relacionada à calagem na neutralização da acidez do solo. Segundo Lopes e Guilherme (2004), em solo mais ácido é maior o conteúdo de alumínio (Al) trocável em valor absoluto e menor os níveis de $\mathrm{Ca}, \mathrm{Mg}$ e $\mathrm{K}$, sendo que possui uma menor soma de bases e maior porcentagem de saturação por alumínio.

A componente PC2, que explica $13,24 \%$ da variação dos dados, apresentou correlação negativa com $\mathrm{H}+\mathrm{Al}$ e positiva com $\mathrm{Ca}$ que também pode ser explicada pelo processo de neutralização do alumínio por esse cátion.

$\mathrm{Na}$ Figura 2 verifica-se a presença de três grupos de variáveis, sendo que um grupo é aquele composto apenas por $\mathrm{H}+\mathrm{Al}$, o qual se correlaciona positivamente com $\mathrm{PC} 1$. O segundo grupo é aquele composto por $\mathrm{pH}, \mathrm{Ca}, \mathrm{V}, \mathrm{SB}$ e $\mathrm{Mg}$, que possuem uma correlação negativa com PC1 e que, por sua vez, estão relacionados negativamente com o $\mathrm{H}+\mathrm{Al}$, de modo que uma calagem que busca o incremento de $\mathrm{V}$ vai contribuir para uma redução dos valores de $\mathrm{H}+\mathrm{Al}$ com um incremento nos valores de $\mathrm{pH}$, de modo a auxiliar na disponibilidade dos nutrientes.

O terceiro grupo é aquele composto por CTC, $\mathrm{K}$ e $\mathrm{MO}$, que possuem correlação positiva com as variáveis do segundo grupo e, consequentemente, negativa com as variáveis do primeiro grupo, com correlação negativa na $\mathrm{PC} 1$, sendo que o incremento no conteúdo de $\mathrm{MO}$, como aquele promovido pela prática do SPD, e K, por aplicação desse nutriente, também contribuem para a redução da acidez no solo.

A componente $\mathrm{PC} 2$ possui correlação negativa com as variáveis dos primeiro grupo $(\mathrm{H}+\mathrm{Al})$, positiva com o segundo grupo e negativa com o terceiro grupo, sendo que a $\mathrm{V} \%$ e o $\mathrm{pH}$ do segundo grupo relacionaram-se negativamente com MO do terceiro grupo. Relações semelhantes foram identificadas em um trabalho que relaciona a fertilidade do solo com a produtividade do morango (ISLABÃO et al., 2012). A relação de V com a matéria orgânica e o $\mathrm{pH}$ pode ser explicado pelo fato de que no processo de mineralização e formação de húmus ocorre a liberação de íons $\mathrm{NO}_{3}^{-}$ e $\mathrm{H}^{+}$, sendo que o ânion $\mathrm{NO}_{3}^{-}$é neutralizado por

Quadro 2. Cargas fatoriais (loadings) dos componentes principais PC1, PC2.

\begin{tabular}{cccccccccc}
\hline & $\mathrm{H}+\mathrm{Al}$ & $\mathrm{Ca}$ & $\mathrm{Mg}$ & $\mathrm{K}$ & $\mathrm{pH}$ & $\mathrm{MO}$ & $\mathrm{SB}$ & $\mathrm{CTC}$ & $\mathrm{V}$ \\
\hline $\mathrm{PC} 1$ & 0,350 & $-0,380$ & $-0,385$ & $-0,228$ & $-0,289$ & $-0,193$ & $-0,395$ & $-0,323$ & $-0,390$ \\
$\mathrm{PC} 2$ & $-0,298$ & 0,194 & 0,000 & $-0,582$ & 0,248 & $-0,625$ & 0,000 & $-0,234$ & 0,153 \\
\hline
\end{tabular}




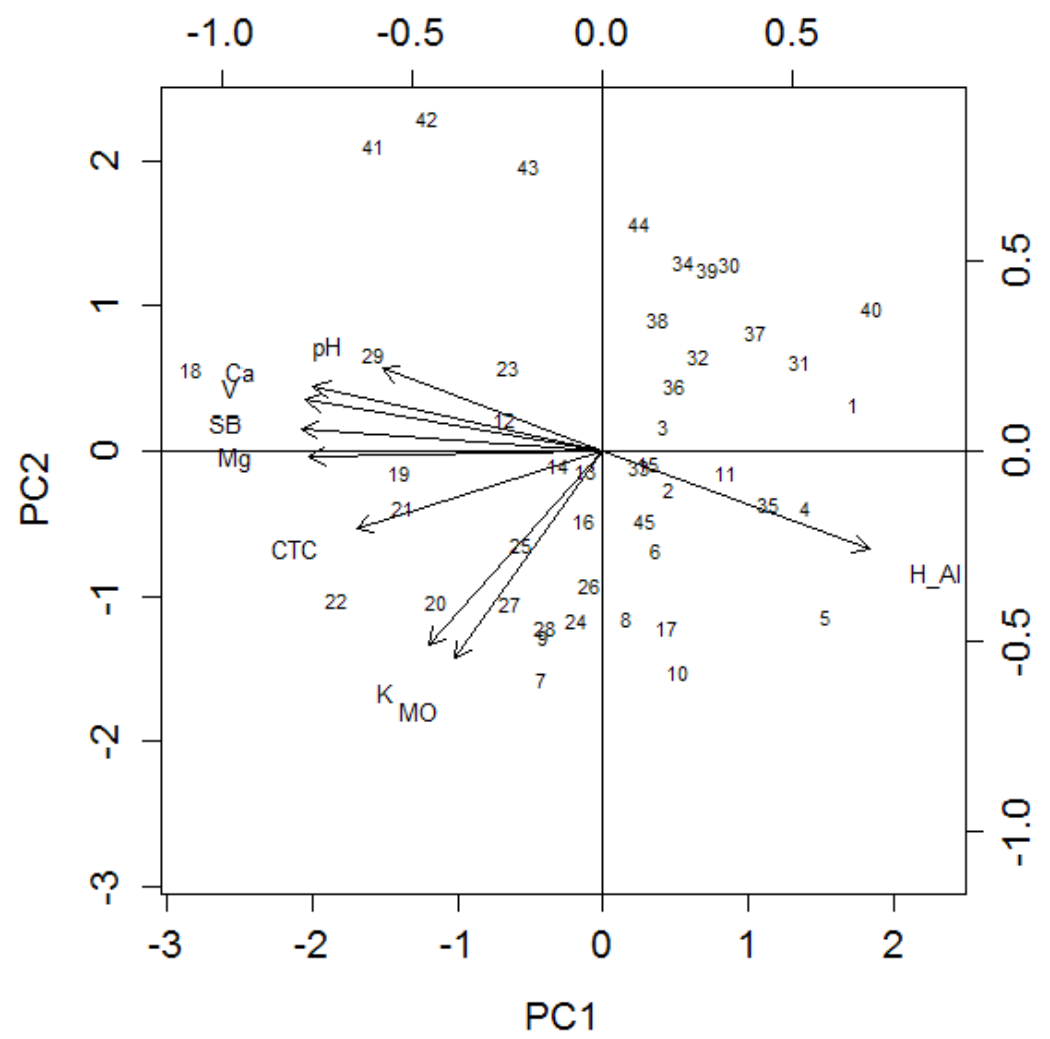

Figura 2. Autovetores das variáveis químicas $\mathrm{H}+\mathrm{Al}, \mathrm{Ca}, \mathrm{Mg}, \mathrm{K}, \mathrm{pH}, \mathrm{SB}, \mathrm{CTC}$ e V.

cátions, como $\mathrm{Ca}$ e $\mathrm{Mg}$, da solução, $\mathrm{V}$ é reduzido, e $\mathrm{H}^{+}$permanece na solução, decrescendo o $\mathrm{pH}$ (MEURER, 2006), o que contribui para a redução de $\mathrm{H}+\mathrm{Al}$.

Os valores para PC1 e PC2 foram estimados em cada um dos 45 elementos amostrais, segundo as ponderações dadas às variáveis

A escolha de um modelo de semivariograma é um procedimento que requer um julgamento baseado na experiência e uma compreensão das limitações matemáticas da função (McBRATNEY; WEBSTER, 1986). O modelo escolhido fornece parâmetros necessários para a krigagem, o interpolador geoestatístico. Neste método, os pesos são atribuídos de acordo com a variabilidade espacial expressa no semivariograma, no entanto, o que torna a krigagem um interpolador ótimo é a maneira como os pesos são distribuídos, não sendo tendencioso, tendo variância mínima e possibilitando que se conheça a variância da estimativa (WEBSTER; OLIVER, 1990).

No Quadro 3 apresentam-se os métodos de estimativa e os parâmetros do modelo gaussiano para as variáveis químicas $\mathrm{H}+\mathrm{Al}, \mathrm{Ca}, \mathrm{Mg}$ e $\mathrm{K}$, $\mathrm{pH}, \mathrm{MO}, \mathrm{SB}, \mathrm{CTC}$ e $\mathrm{V}$ e para os $\mathrm{PC} 1$ e $\mathrm{PC} 2$, utilizados para os respectivos semivariogramas experimentais, obtidos com o estimador de Matheron, com um mínimo de 30 pares de pontos para cada semivariância.

O efeito pepita relativo (EPR) foi utilizado para definir as classes de dependência espacial dos atributos (CAMBARDELLA et al.,1994). O EPR acima de $75 \%$ indica que a dependência espacial é fraca; EPR maior ou igual a $25 \%$ e menor ou igual a $75 \%$ a dependência espacial é moderada; EPR menor do que $25 \%$ a dependência espacial é forte. Os valores de EPR para P, PC1 e PC2 indicaram que possuem forte dependência espacial.

$\mathrm{Na}$ Figura 3 estão apresentados os mapas de superfície com os valores observados dos 45 elementos amostrais para as variáveis $\mathrm{H}+\mathrm{Al}, \mathrm{Ca}$, $\mathrm{Mg}, \mathrm{K}, \mathrm{SB}, \mathrm{CTC}$ total, $\mathrm{V}, \mathrm{pH}$ e MO com o modelo gaussiano, método de estimação e parâmetros apresentados no Quadro 4. Os mapas de superfícies foram obtidos com a interpolação de krigagem.

$\mathrm{O}$ potássio (Figura 3D) apresentou teores classificados como bom e muito bom (EMBRAPA, 2013), os quais podem contribuir significativamente para a produtividade de grãos, como na cultura do milho, e, associado à elevada mobilidade desse elemento no solo, pode contribuir 
Quadro 3. Estimativas dos parâmetros do modelo Gaussiano para os semivariogramas experimentais das variáveis químicas e seus componentes principais PC1 e PC2.

\begin{tabular}{lcccccc}
\hline & Método & $\varphi_{1}$ & $\varphi_{2}$ & $\varphi_{3}$ & $\varphi_{4}$ & EPR (\%) \\
\hline $\mathrm{H}+\mathrm{Al}$ & OLS & 0,000 & 0,273 & 150,986 & 261,330 & - \\
$\mathrm{Ca}$ & OLS & 0,000 & 0,232 & 170,413 & 294,955 & - \\
$\mathrm{Mg}$ & OLS & 0,000 & 0,126 & 201,849 & 349,363 & - \\
$\mathrm{K}$ & OLS & 0,000 & 0,0038 & 185,572 & 321,192 & - \\
$\mathrm{pH}$ & OLS & 0,000 & 0.0207 & 189.5460 & 328.0697 & - \\
$\mathrm{MO}$ & OLS & 0.000 & 0.057 & 240.000 & 415.4 & - \\
$\mathrm{SB}$ & OLS & 0.000 & 0.724 & 183.868 & 318.2 & - \\
$\mathrm{CTC}$ & OLS & 0.000 & 0.216 & 182.385 & 315.7 & - \\
$\mathrm{V} \%$ & OLS & 2.217 & 23.256 & 183.777 & 318.1 & 8,703 \\
PC1 & OLS & 0.728 & 7.997 & 188.601 & 326.4 & 8,343 \\
PC2 & OLS & 0.0000 & 0.8005 & 158.788 & 274.833 & - \\
\hline
\end{tabular}

$\varphi_{1}$ : efeito pepita; $\varphi_{2}$ : contribuição; $\varphi_{3}=g\left(\varphi_{4}\right)$ : função do alcance; $\varphi_{4}$ : alcance; EPR: efeito pepita relativo sendo $E P R=\left(\frac{\varphi_{1}}{\varphi_{1}+\varphi_{2}}\right)-100 ;$ OLS: mínimos quadrados ordinários; WLS: mínimos quadrados ponderados.

significativamente para o elevado coeficiente de variação da produtividade de grãos (RAIJ et al., 1997). As regiões com altos teores de K podem promover alterações na absorção de $\mathrm{Ca}$ e $\mathrm{Mg}$ (NOGARA NETO et al., 2011), de modo que pode contribuir na lixiviação desses dois cátions no solo, em razão da competição por sítios do complexo de troca de cátions.

Os teores de cálcio (Figura 3B), classificados como médio e alto (EMBRAPA, 2013), e de magnésio (Figura 3C), classificados como alto, contribuem para a produtividade de grãos. Entre as várias limitações à produção de grãos, decorrentes da deficiência desses elementos, pode-se considerar a acidez (RAIJ et al.,1997). A acidez potencial (Figura 3A), possui os valores classificados como alto e muito alto (RIBEIRO et al., 1999), os quais são limitantes para o desenvolvimento de culturas.

Segundo Raij et al. (1997), regiões em que a saturação por bases é classificada como média a alta contribuem para a limitação provocada pela acidez do solo, mesmo quando os valores de cálcio sejam classificados como baixo e médio. As Figuras 3A e 3E permitem uma visualização de regiões com valores mais elevados de acidez em regiões correspondentes, em que a saturação por base é menor, porém ainda classificada como de média a alta (RIBEIRO, et al., 1999).

As Figuras 4A e 4B apresentam os mapas de superfície para os escores de PC1 e PC2, considerando os pesos, provenientes da análise de componentes principais, nos 45 elementos amostrais das variáveis químicas observadas, $\mathrm{H}+\mathrm{Al}, \mathrm{Ca}, \mathrm{Mg}, \mathrm{K}, \mathrm{SB}, \mathrm{CTC}, \mathrm{V}, \mathrm{pH}$ e MO . Os mapas de superfícies foram elaborados por krigagem utilizando os parâmetros obtidos de modelos gaussianos ajustados pelo método dos mínimos quadrados ordinários.

A Figura 4A possui regiões em preto, que caracterizam os maiores valores dos escores, de modo que nessas regiões observa-se maior acidez no solo (escores de -24,29 a -21,58), comparativamente com as demais regiões nessa superfície, já que as contribuições positivas são atribuídas a $\mathrm{H}+\mathrm{Al}$ e negativas as demais variáveis. As regiões de maior acidez correspondem aquelas com valores mais baixos de cátions, como $\mathrm{Ca} \mathrm{e}$ $\mathrm{Mg}$, que neutralizam o $\mathrm{H}+\mathrm{Al}$. Os valores de $\mathrm{SB}$, $\mathrm{V}, \mathrm{K}$ e pH também encontra-se mais baixos. Por outro lado, os menores valores dos escores $(-35,15$ a $-32,43)$ de PC1 indicam as regiões com menor acidez e corresponde com aquelas de maiores valores de $\mathrm{Ca}, \mathrm{Mg}, \mathrm{SB}, \mathrm{V}, \mathrm{K}$ e $\mathrm{pH}$.

Assim, nas Figuras 1A e 1B, ficam indicadas, de modo multivariado, as regiões que requerem operações de correção de acidez no solo e que devem ser realizadas a taxas variadas de modo a incrementar a fertilidade da área.

\section{REVENG}




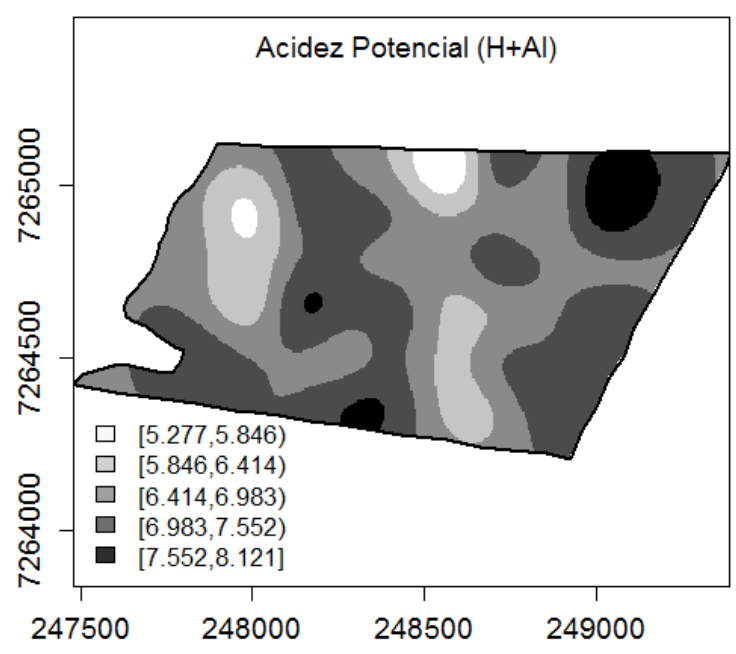

(A)

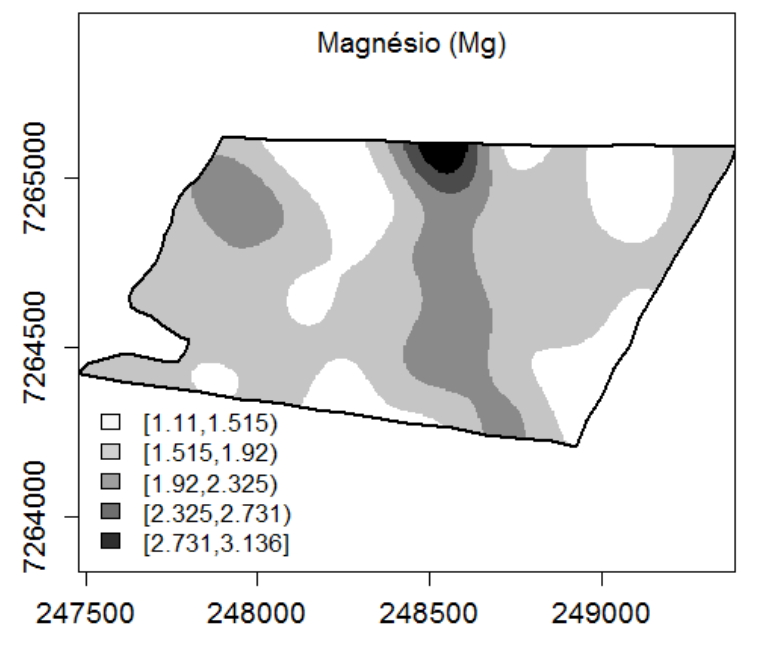

(C)

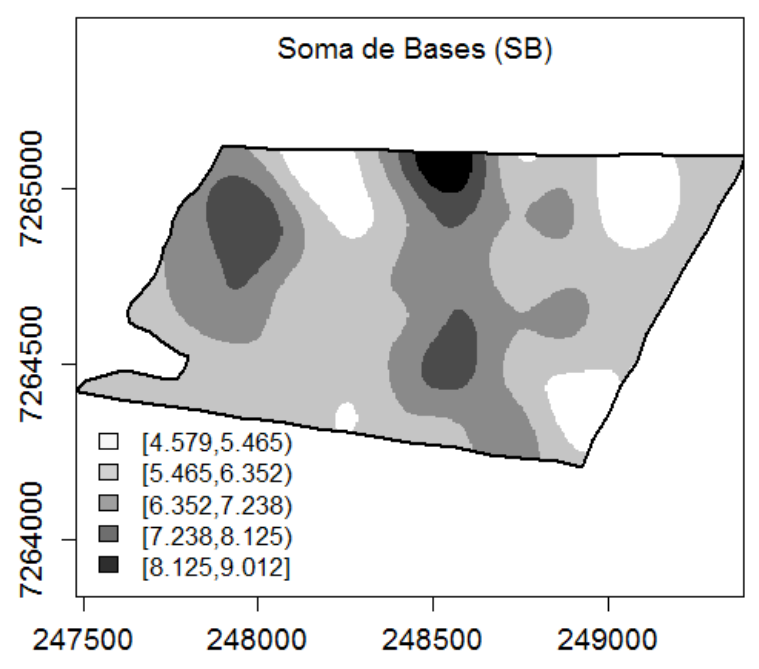

(E)

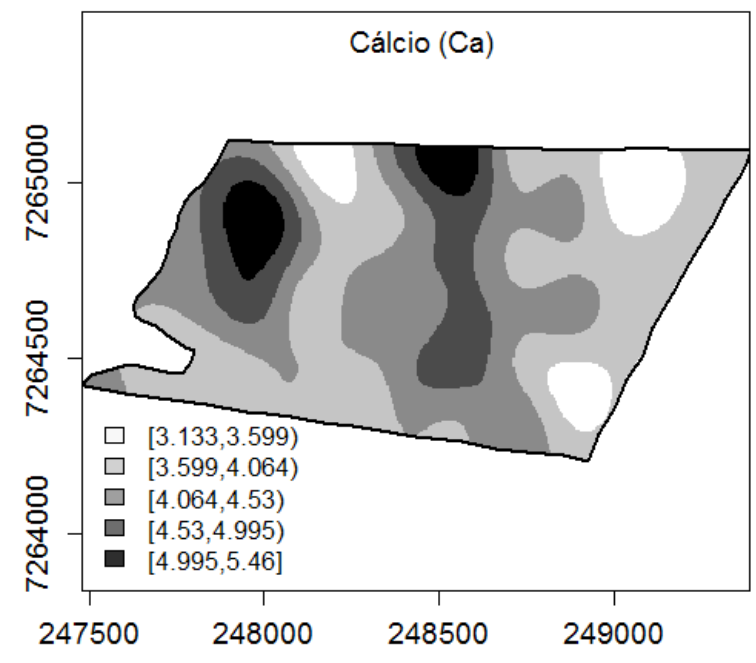

(B)

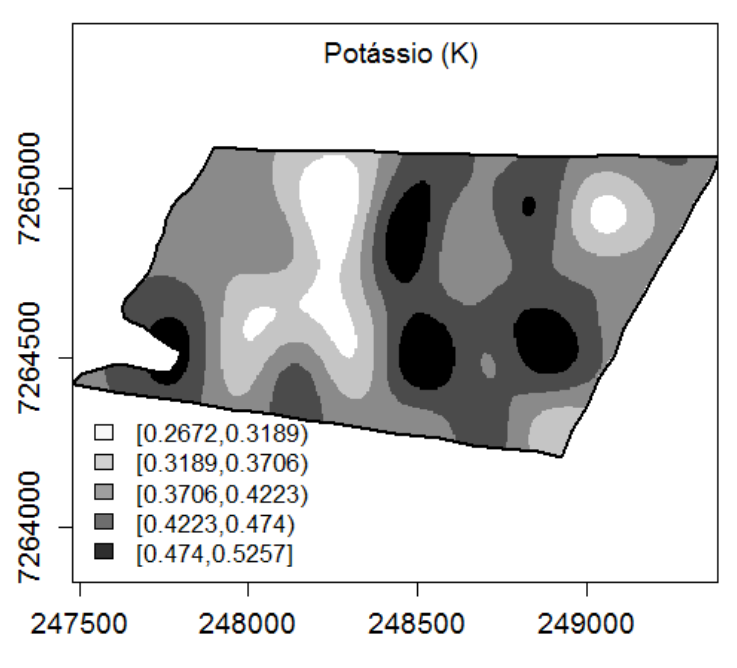

(D)

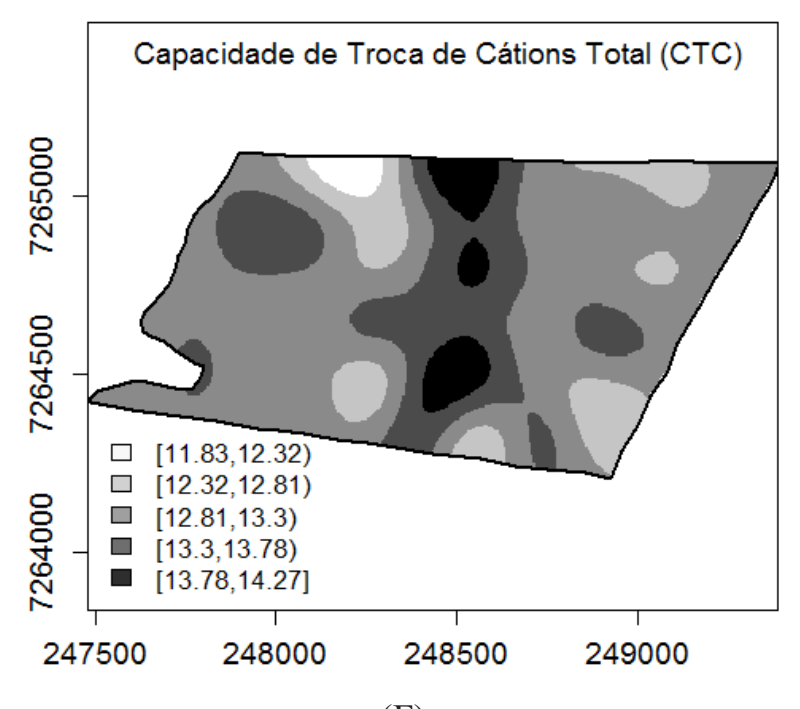

(F) 


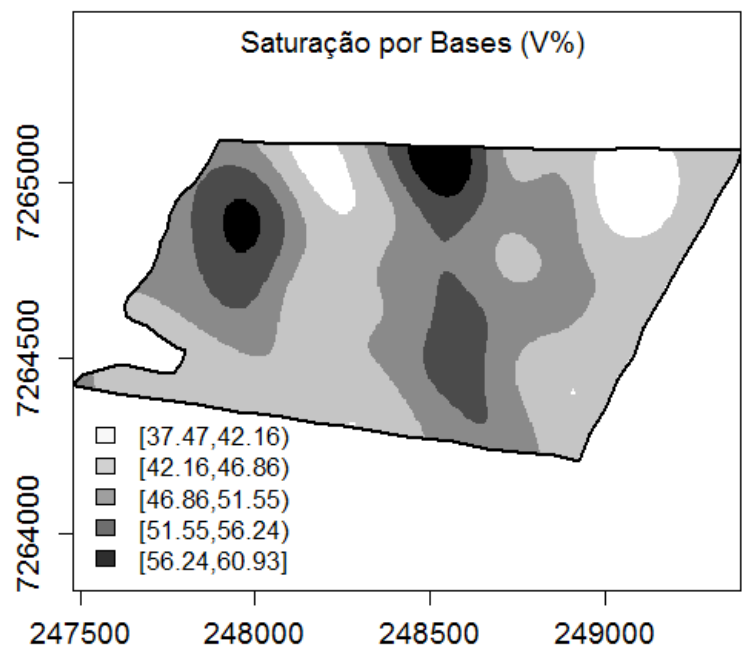

$(\mathrm{G})$

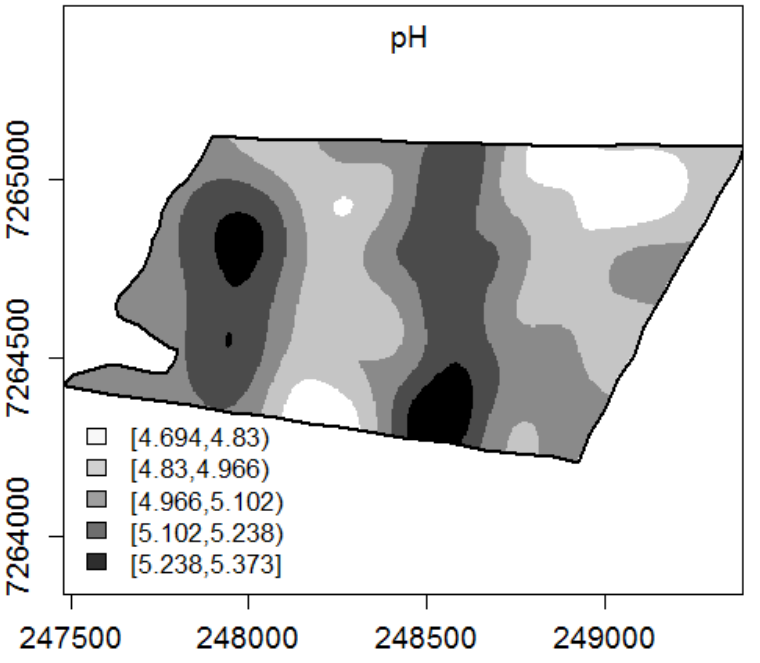

$(\mathrm{H})$

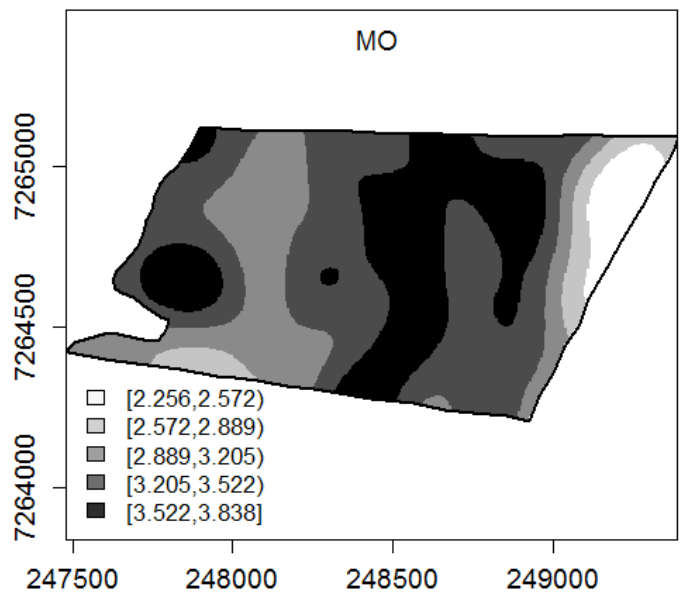

(I)

Figura 3. Mapas temáticos das variáveis químicas do solo $\mathrm{H}+\mathrm{Al}, \mathrm{Ca} . \mathrm{Mg}, \mathrm{K}, \mathrm{SB}, \mathrm{CTC}, \mathrm{V}, \mathrm{pH}$ e MO.

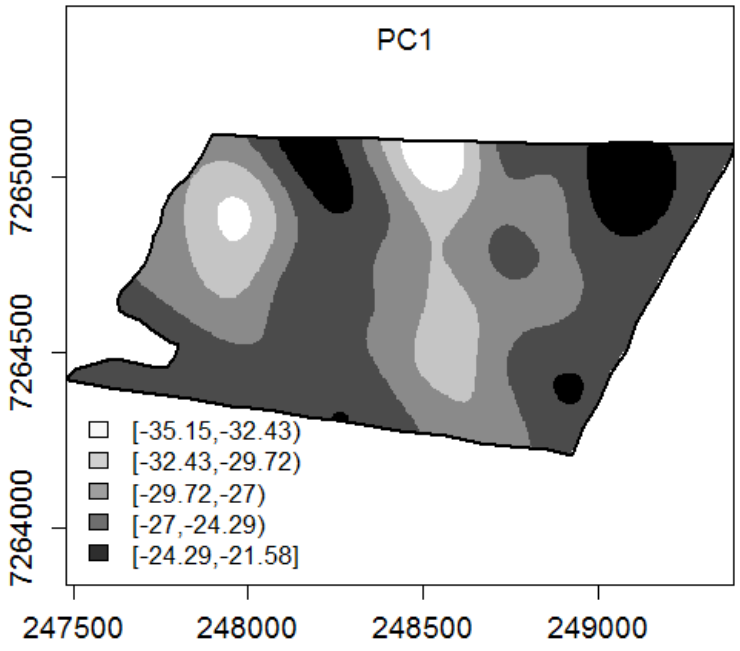

(A)

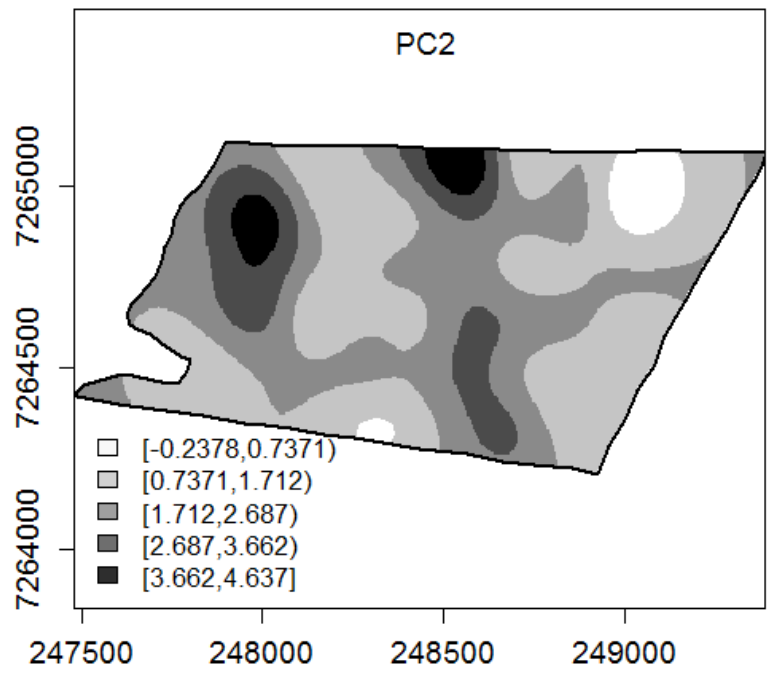

(B)

Figura 4. Mapas de superfícies dos escores das componentes PC1 e PC2.

\section{REVENG




\section{CONCLUSÕES}

- As propriedades relacionadas com a fertilidade do solo observadas apresentaram dependência espacial na área estudada;

- A análise de componentes principais permitiu o agrupamento das propriedades relacionadas com a acidez do solo;

- A construção de mapas das propriedades do solo e dos escores das componentes principais permitiu interpretar espacialmente relações de atributos da fertilidade do solo, de modo que as áreas acidificadas corresponderam com aquelas com deficiência de cálcio e magnésio utilizado na neutralização da acidez;

- A área em estudo pode ser dividida em três subregiões, as quais direcionam as amostragens convencionais para monitoramento dos níveis de nutrientes do solo.

\section{REFERÊNCIAS BIBLIOGRÁFICAS}

ADHIKARI, P.; SHUKLA, M.K.; MEXAL, J.G.; SHARMA, P. Assessment of the soil physical and chemical properties of desert soils irrigated with treated wastewater using principal component analysis. Soil Science, v.176, n.7, p.356-366, 2011.

AMADO, T.J.C; PONTELLI, C.B.; SANTI, A.L.; VIANA, J.H.M.; SULZBACH, L.A.S. Variabilidade espacial e temporal da produtividade de culturas sob sistema plantio direto. Pesquisa Agropecuária Brasileira, v.42, n.08, p. 1101-1110, 2007.

ARTHUR, T.J.; TONKIN, J.H.B. Testando o vigor da semente. Informativo Associação Brasileira de Tecnologia de Sementes, Londrina, v.1, n.3, p.38-42, 1991.

CAMBARDELLA, C.A.; MOORMAN, T.B.; NOVAK, J.M.; PARKIN, T.B.; KARLEN, D.L.; TURCO, R.F.; KONOPKA, A.E. Field-scale variability of soil properties in central Iowa soils. Soil Science Society America Journal, Madison, v.58, n.5, p.1501-1511, 1994.

CELIK, A.; BOYDAS, M.G.; ALTIKAT, S.A comparison of an experimental plow with a moldboard and a disk plow on the soil. Applied Engineering in Agriculture, v.27, n.2, p.185-192, 2011.

CORÁ, J.E.; ARAUJO, A.V.; PEREIRA, G.T.; BERALDO, J.M.G.. Variabilidade espacial de atributos do solo para adoção do sistema de agricultura de precisão na cultura de cana-de-açúcar. Revista Brasileira de Ciência do Solo. v.28, n.6, pp. 10131021, 2004.

EMPRESA BRASILEIRA DE PESQUISA AGROPECUÁRIA-EMBRAPA. Centro Nacional de Pesquisa de Solos. Sistema brasileiro de classificação de solos. 2.ed. Rio de Janeiro, 2006. 306p.

EMPRESA BRASILEIRA DE PESQUISA AGROPECUÁRIA. Conceitos de fertilidade do solo e manejo adequado para as regiões tropicais. Campinas, SP, 2010. 28p.

EMPRESA BRASILEIRA DE PESQUISA AGROPECUÁRIA. Sistemas de Produção: Tecnologias de Produção de Soja - Região Central do Brasil 2012 e 2013. Londrina, PR: Embrapa Soja, 2011. 262p.

FERREIRA, D. F. Estatística Multivariada. 1.ed. Lavras: Ed. UFLA, 2008. 651p.

GOMES, P. Curso de Estatística Experimental. 14 ed. Piracicaba: Degaspari, 2000. 477p.

LOPES, S.A.; GUILHERME, L.R.G. Interpretação De Análise De Solo: Conceitos E Aplicações. São Paulo, Associação Nacional para Difusão de Adubos - ANDA, 2004. 50p.

MANDAL, U.K.; WARRINGTON, D.N.; BHARDWAJ, A.K.; BAR-TAL, A.; KAUTSKY, L.; MINZ, D.; LEVY, G.J. Evaluating impact of irrigation water quality on a calcareous clay soil using principal component analysis. Geoderma, v.144, p.189-197, 2008.

MATHERON, G. Principles of geoestatistics. Economic Geology, Littleton, v.58, n.8, p.1246-1266, 1963. 
McBRATNEY, A.; WEBSTER, R. Choosing functions for semi-variograms of soil properties and fitting them to sample estimates. Journal of Soil Science, Oxford, v.37, n.4, p.617-639, 1986.

NOGARA NETO, F.; ROLOFF, G.; DIECKOW, J.; MOTTA, A.C.V. Atributos de solo e cultura espacialmente distribuídos relacionados ao rendimento do milho. Revista Brasileira de Ciência do Solo, v.35, n.3, p.1025-1036, 2011.

JOHNSON, R.A.; WICHERN D.W.. Applied Multivariate Statistical Analysis. $6^{\mathrm{a}}$ ed. Pearson Education, Inc., Upper Saddle River, NJ, 2007. $767 \mathrm{p}$.

RAIJ, B. Van; CANTARELLA, H.; QUAGGIO, J.A.; FURLANI, A.M.C. Recomendações de adubação e calagem para o Estado de São Paulo. 2.ed. Campinas: Instituo Agronômico de Campinas (IAC), 1997. 285p.

RIBEIRO, A.C.; GUIMARÃES, P.T.G.; ALVAREZ V., V.H. Recomendação para o uso de corretivos e fertilizantes em Minas Gerais: 5a aproximação. Viçosa, MG: Comissão de Fertilidade do Solo do Estado de Minas Gerais, 1999. 359p.

RIBEIRO JÚNIOR, P.J. Métodos geoestatísticos no estudo da variabilidade espacial de parâmetros do solo. 1995. 99f. Dissertação (Mestrado em Agronomia) - Escola superior de Agricultura "Luiz de Queiroz", Universidade de São Paulo, Piracicaba, 1995.

SILVA, S.A. et al. Variabilidade espacial de atributos químicos de um Latossolo Vermelho-Amarelo húmico cultivado com café. Revista Brasileira de Ciência do Solo, v.34, p.15-22, 2010.

THOMAS, A.L.; COSTA, J.A.; PIRES, J.L. Rendimento de grãos de soja afetado pelo espaçamento entre linhas e fertilidade do solo. Ciência Rural, Santa Maria, v.28, n.4, p.543-546, 1998.

TORMENA， C.A.; ROLOFF， G.; SÁ, J.C.M. Propriedades físicas do solo sob plantio direto influenciadas por calagem, preparo inicial e tráfego. Revista Brasileira de Ciência do Solo, Campinas, v.22. p.301-309, 1998.

VALLADARES, G.S.; GOMES, E.G.; MELLO, J.C.C.B.S.; PEREIRA, M.G.; ANJOS, H.C.; EBELING, A.G.; BENITES, V.M. Análise dos componentes principais e métodos multicritério ordinais no estudo de Organossolos e solos afins. Revista Brasileira de Ciência do Solo, v.32, n.1 p.285-296, 2008.

WARRICK, A.W.; NIELSEN, D.R. Spatial variability of soil physical properties in the field. In: HILLEL, D., ed. Applications of soil physics. New York, Academic Press, 1980. 350p.

WATANABE, R.T. et al. Produtividade da cultura da soja em função da densidade populacional e da porcentagem de cátions ( $\mathrm{Ca}, \mathrm{Mg}$ e $\mathrm{K})$ no complexo sortivo do solo. Semina: Ciências Agrárias, v.26, n.04, p.477-484, 2005.

WEBSTER, R.; OLIVER, M.A. Statistical Methods in Soil and Land Resource Survey. Oxford University Press, Oxford, 1990. 316p. 